\title{
Immediate versus deferred treatment for early prostatic cancer
}

\author{
P.H. Smith and T.G. Armitage \\ Department of Urology, St. James's University Hospital, Beckett Street, Leeds LS9 7TF, UK.
}

\begin{abstract}
Summary: The diagnosis of early (non-metastatic) prostatic cancer is dependent upon the patient presenting himself with symptoms or for routine rectal examination or ultrasound screening. Diagnosis in the absence of symptoms is more common outside the United Kingdom and as a result the opportunity to treat such patients occurs more commonly in other countries.

The treatment options for the different stages are reviewed and the evidence for their effectiveness is considered.

A review of the literature yields conflicting evidence of the efficacy of and the necessity to institute early therapy. This basic gap in our current knowledge should be filled when the results of the existing Medical Research Council (MRC) clinical trial of immediate and deferred orchidectomy are available.
\end{abstract}

\section{Introduction}

The choice of immediate or deferred therapy for patients with early (non-metastatic) prostatic cancer is dependent upon two factors - diagnosis of the condition at an early stage and a conviction that effective therapy is available.

In the United Kingdom there is no generally available screening programme for the detection of asymptomatic prostatic cancer at a time when it might be amenable to all treatment options including radical surgical excision. The earlier stages of disease will inevitably be found more commonly in the younger patients. If it can be established that early therapy influences the survival early diagnosis will be mandatory.

Once the diagnosis has been made treatment can be given. That which is chosen will be determined in part by the professional training and inclination of the doctor in charge of the patient and in part by a general perception of the effectiveness of the alternative therapeutic regimens. The major options are shown in Table I and include deferred treatment, radical surgery, radiotherapy and hormonal therapy. At the present time chemotherapy is not a viable option as it is not yet sufficiently effective.

From Table I it can be seen that none of the options is ideal. The choice of compromise will be determined by the hazard which is acceptable to the patient and the benefit that he may expect from the therapy.

Correspondence: P.H. Smith F.R.C.S.

Received: 16 June 1987
Only when the choice of treatment has been decided does the question of its timing become of great relevance. This paper analyses the evidence in favour of the different forms of treatment and considers the possible place of deferred therapy.

The basis of effective cancer control is to excise the primary tumour whilst it is still confined or to destroy it in situ by irradiation. This ideal has led to the use of radical prostatectomy and radical radiotherapy in lesions confined to the prostate. If radical surgery or radiotherapy is not considered appropriate hormonal treatment may be given.

\section{Diagnosis}

Assessment of the patient's primary tumour by digital palpation, ultrasound and biopsy will show that he has either: (1) latent cancer with focal or diffuse histological evidence of malignant disease; (2) a nodule localized to the prostate (Category $\mathrm{T} 1$ or $\mathrm{T} 2$ disease) or (3) extra-capsular but non-metastatic disease (Category T3).

The further diagnostic evaluation of such patients includes histological or cytological confirmation of diagnosis, determination that the alkaline and acid phosphatase levels are normal and confirmation that the bone scan shows no abnormal hot spots. If radical surgery is contemplated it is also necessary to know that the lymph nodes are free from tumour. Nodal assessment may be undertaken by lymphangiography, 
Table I Treatment options in prostatic cancer

\begin{tabular}{|c|c|c|}
\hline Treatment options & Advantage & Hazard \\
\hline Deferred treatment & $\begin{array}{l}\text { No change in quality } \\
\text { of life }\end{array}$ & Progression \\
\hline Radical surgery & $\begin{array}{l}\text { Removal of the } \\
\text { primary tumour }\end{array}$ & $\begin{array}{l}\text { Impotence and } \\
\text { incontinence }\end{array}$ \\
\hline Radiotherapy & $\begin{array}{l}\text { 'Destruction' of the } \\
\text { primary tumour }\end{array}$ & $\begin{array}{l}\text { Rectal-protitis } \\
\quad \text {-stenosis } \\
\text { Vesical-haematuria } \\
\text { Urethral-stricture }\end{array}$ \\
\hline Hormonal & $\begin{array}{l}\text { Suppression of } \\
\text { tumour growth }\end{array}$ & $\begin{array}{l}\text { Loss of libido } \\
\text { Impotence } \\
\text { Cardiovascular hazard }\end{array}$ \\
\hline
\end{tabular}

fine needle aspiration, or by lymphadenectomy.

\section{Principles of management}

Management of these three groups of patients in the absence of metastases will depend to some extent on the patient's age and general condition and also upon the histological grade of the tumour.

Latent cancer is found in up to $30 \%$ of men aged 70 years or more ${ }^{1}$ and like cancer which is clinically evident but non-metastatic, often progresses very slowly without treatment. Even in patients with disease clinically beyond the prostatic capsule only $50 \%$ in one series developed evidence of metastases after 5 years of observation. ${ }^{2}$

Age is an important factor in patients with localized disease as, in the elderly, up to two thirds of deaths within 5 years are not due to prostate cancer ${ }^{2}$ whilst in the younger patient most will eventually die of, rather than with, their tumours. ${ }^{3}$

The slow rate of progression in many patients and the recognition that death may occur from some cause other than prostatic cancer is likely to lead one towards an expectant approach in the elderly patient but to a more active role in the younger man.

The grade of tumour is also of marked prognostic value. All studies show impaired survival for those with poorly differentiated tumours and active treatment is more commonly given to such patients. This approach reflects the concern of the doctor for his patient and an acknowledgement of the risk which the patient faces. There is little evidence so far, however, that any treatment for the patient with a poorly differentiated tumour is likely to be curative.

An attempt is made in the following paragraphs to evaluate the effectiveness of the three major forms of therapy and to analyse their relevance to the patient with latent cancer, a nodule localized to the prostate and a tumour which has already spread beyond the capsule but which shows no clinical evidence of metastases.

\section{Immediate treatment}

If the tumour is confined to the prostate, removal of the gland with its capsule should cure the patient. A failure to do so implies the possibility of tumour dissemination at the time of the operation or of preexisting microscopic foci of disease.

Since the operation was introduced by Young in 1917 it has been used with enthusiasm in many centres but has yet to become routine medical practice. Until recent years radical prostatectomy was followed by impotence in nearly all patients and by incontinence in up to $20 \%$. However the introduction of the nervesparing technique by Walsh et al. ${ }^{4}$ has changed the outlook for the patient and thus the attitude of the surgeon.

In the studies carried out by the Veterans Administration Cooperative Urological Research Group (VACURG) radical prostatectomy was undertaken in the first study with or without oestrogen therapy and in the second study radical prostatectomy was compared against placebo treatment. In analysis up to 10 years no survival advantage was demonstrated for those with latent cancer or with nodules confined to the prostate. ${ }^{5}$ Of the deaths in the patients randomized to radical prostatectomy or to placebo there were only 4 from prostatic cancer in 111 patients up to 8 years after operation. Of these, 3 occurred in the patients treated by radical operation. There was no significant difference in the overall survival of these 111 patients 61 with stage 1 disease (latent cancer) and 50 with stage 2 disease (a localized nodule within the prostatic capsule).

This argument against radical surgery takes no account of the difference between focal and diffuse latent disease - a concept which has only been widely 
recognized within the last 10 years. Since that report, emphasis has been placed upon the concept of 'diffuse' and 'focal' Stage I disease. In 1974 Correa et al. ${ }^{6}$ reported 47 Stage 1 patients, eight of whom had diffuse and 39 focal disease. Treatment was by diethylstilboestrol (DES) $1 \mathrm{mg} /$ day (23 patients), radical prostatectomy (2 patients) and no therapy (22 patients). Diffuse carcinoma was found in eight patients, five of whom $(62 \%)$ showed progression of tumour with two deaths. Only three of the 39 patients with focal disease experienced progression, and none died of prostatic cancer. Treatment was evenly distributed within the two groups, except that both patients undergoing radical prostatectomy had focal disease. These authors believe that treatment in both groups should be delayed until clinically detectable disease appears, since with either treatment survival is very similar to that of the normal male population of a similar age. It may, however, be more logical to treat all patients with diffuse disease in view of the known high rate of progression, this being seen also in $16 \%$ of patients with focal (stage A1) disease with a follow-up of at least 8 years. $^{7}$

In favour of total prostatectomy for localized prostatic cancer is the article by Gibbons et al. ${ }^{8}$ who, in a series of 215 consecutive patients treated with localized prostatic cancer, of whom 195 had clinical Stage B disease, 16 Stage A disease and 2 Stage C disease, noted an overall survival in 213 evaluable patients of $55 \%$ at 15 years; $48 \%$ of the patients were clinically free of disease.

All these patients had an expected 15 year survival and underwent radical perineal prostatectomy without adjuvant hormone therapy unless disease recurred. There were no operative deaths. Gibbons draws attention to other patients with localized disease in whom deferred therapy, endocrine treatment with or without TUR (transurethral resection), retroperitoneal pelvic lymphadenectomy plus iodine125 , retroperitoneal lymphadenectomy, gold-198 and radiotherapy, or external heam radiotherapy had all shown inferior results at 5 and 10 years. In the majority of studies results were not available at 15 years and where they were, were less satisfactory. The overall survival obtained by Gibbons et al ${ }^{8}$ was virtually identical to the expected survival rate.

It is of interest that only 11 patients in this series had very poorly differentiated tumours since Elder et al. ${ }^{9}$ who reviewed 53 patients undergoing radical prostatectomy for clinical Stage B2 prostatic cancer and who commented on the results of a series of patients with clinical Stage B1 disease undergoing the same operation, noted that no patient with a poorly differentiated tumour survived 15 years. This leaves unresolved the question as to whether radical prostatectomy is suitable for all patients with cancer which is clinically localized to the prostate since the patient with poorly differentiated disease does not survive and some patients $(25 \%$ of clinical Stage B disease in the series of Gibbons et al.) need subsequent hormonal therapy because of recurrent disease.

\section{Radiotherapy}

Radiotherapy by external beam irradiation or by interstitial implantation has been used extensively in patients with localized prostatic cancer. Several large series have been reported..$^{10,11,12}$ All comment upon the good results obtained when irradiation is used for patients with well or moderately differentiated disease but none has demonstrated good survival figures for patients with poorly differentiated lesions.

In recent years Paulson and the National UroOncology Research Group have undertaken four separate studies. ${ }^{13,14,15}$ In the first, patients with nodepositive disease were treated on a non-randomized basis by radical prostatectomy, external beam radiation therapy or delayed androgen deprivation. In the 11 patients treated by radical operation and the 33 by radiotherapy or delayed androgen deprivation half the patients in each group progressed within 18 months of treatment suggesting that the presence of extension of the tumour to the lymph nodes identified a lesion which had spread beyond the confines of local therapy.

In two subsequent randomized studies patients with multi-focal latent cancer (A2 disease) or clinically evident nodules contained within the prostate were treated by radical operation or radiotherapy if nodenegative (Study 2) and by extended field radiation therapy or delayed hormonal therapy if node-positive or if the tumour extended beyond the prostatic capsule (Studies 3 and 4, Table II).

In a further group of patients with Category T3 disease (extending beyond the prostatic capsule) 73 patients were randomized to receive either external beam radiation or delayed androgen ablation.

Although the total number of patients in these studies was small and even though the numbers in each group were not identical despite randomization the authors felt able to conclude that: (a) radiotherapy at diagnosis was less effective than radical operation in delaying progression in those with node-negative disease limited to the prostatic capsule; (b) delayed hormonal therapy was as effective as external radiotherapy in node-positive patients whose primary tumour was limited to the prostatic capsule, and that (c) hormonal therapy and radiotherapy appeared to be equally effective in delaying progression in patients with node-negative disease whose primary lesion was beyond the prostatic capsule.

The fact that the progression rate was more rapid in those receiving radiotherapy than in those treated by 
Table II Schema of randomized trials by the National Uro-Oncology Research Group ${ }^{13-15}$

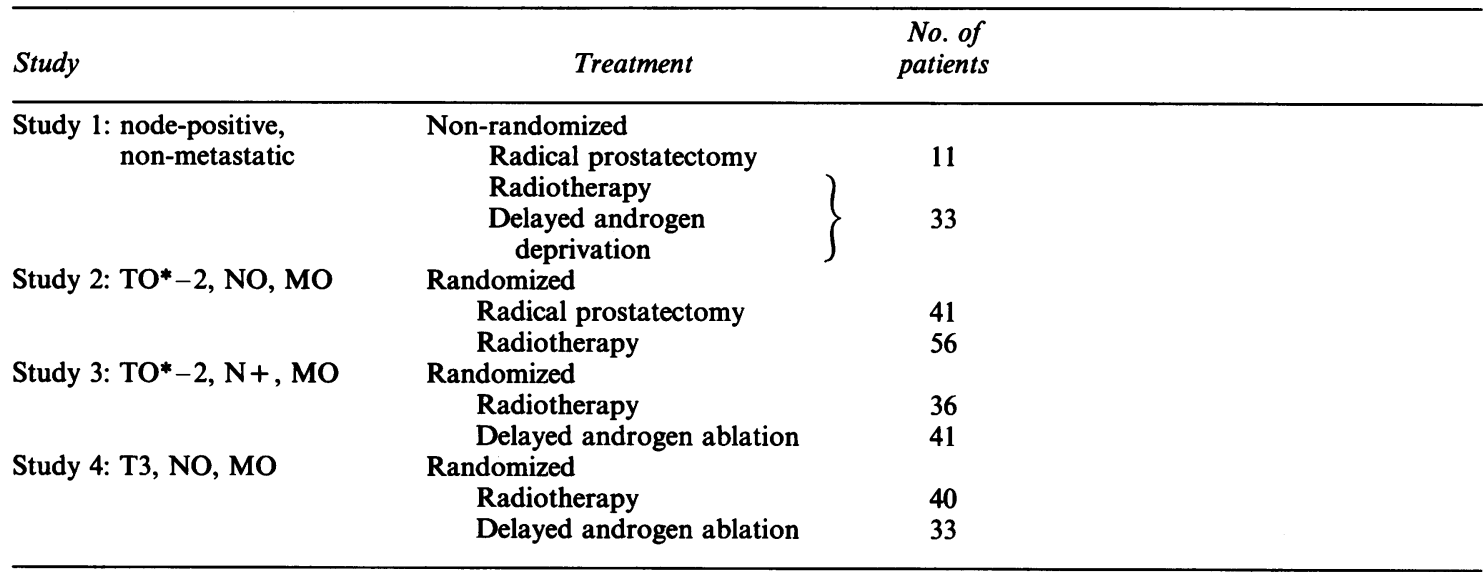

*Patients with focal disease only when excluded.

The categories in Studies 2 to 4 are those of the TNM system (Harmer, M.H. TNM classification of malignant tumours. 3rd ed. Geneva International Union Against Cancer, 1978.)

radical operation and that the progression rates in patients receiving radiotherapy or delayed hormonal therapy were equal casts doubt on the value of any external radiotherapy especially when one considers that the patients randomized to deferred hormonal treatment were receiving no treatment at all from the time of diagnosis to the time of progression.

\section{Hormonal therapy}

The classical work of the Veterans Administration Cooperative Urological Research Group ${ }^{2,16,17,18}$ and the more recent results of the Urological Group of the EORTC (European Organisation for Research on the Treatment of Cancer) ${ }^{19,20}$ have clearly demonstrated that treatment with diethylstilboestrol (DES) or orchidectomy will relieve symptoms in the majority of patients and will induce a remission of metastatic and local disease in approximately $30 \%$ of patients. None of these studies has demonstrated that stilboestrol, orchidectomy, estramustine phosphate, cyproterone acetate or medroxyprogesterone acetate in the doses used are likely to prolong survival.

\section{Deferred therapy}

It will be recollected that in the VACURG studies patients were randomized between placebo as first treatment and 'active' therapy. In the studies of the EORTC Urological Group all patients were treated. The overall survival figures in the EORTC studies are, however, no different from those obtained in the
VACURG studies and have not contributed to the debate upon the necessity for immediate treatment in the absence of symptoms in patients with localized or with metastatic disease.

This debate is not new and Scott ${ }^{21}$ observed in the 1950 s 'I am one of those who believe that hormonal treatment in prostatic cancer should be reserved for the patient who has metastases which are causing pain.' Paulson ${ }^{22}$ in an analysis of his own work concluded that 'multi-modal therapy consisting of pelvic lymphadenectomy with or without external beam radiotherapy has no benefit over surgery or no treatment.'

Although the majority of the profession incline towards treatment at the time of diagnosis the arguments in favour of such a course of action (especially in patients with no symptoms) have never been conclusively proved. If anything a critical and logical interpretation of previous published results from randomized studies argues against immediate treatment unless for the relief of symptoms. This point has recently been highlighted by Fair. ${ }^{23}$

Because of this continuing debate the Prostatic Cancer Subgroup of the Urological Working Party of the Medical Research Council considered the question of immediate versus deferred therapy. The majority of the urologists involved regarded orchidectomy as the standard and safest form of hormonal treatment and agreed to a randomized study of immediate orchidectomy versus orchidectomy deferred until such time as it proved to be indicated. This study is being coordinated by Mr David Kirk of Glasgow and has so far recruited over 350 patients. Those actively involved are anxious to increase the size of the trial since, like 
Professor Stamey, ${ }^{24}$ they believe that it is important to attempt to resolve the question of the importance or otherwise of initiating treatment at the time of first diagnosis.

For the moment it is only possible to say that, despite considerable effort over forty years, the results of randomized clinical trials do not show that any treatment has yet been proved to be superior to diethylstilboestrol or bilateral orchidectomy for the management of metastatic prostatic cancer and that it is as yet quite uncertain whether treatment in the absence of symptoms is best initiated at the time of diagnosis or only upon subsequent objective progression or the development of symptoms which demand relief.

In taking a decision to treat, one must inevitably accept that the patient's quality of life will be affected by the treatment and understand that radical surgery and radical radiotherapy may both be followed by long-term complications which include incontinence, impotence, bladder contracture, urethral stricture and irradiation proctitis. It is by no means certain that radiotherapy is effective in contrölling disease

\section{References}

1. Wynder, E.L., Mabuchi, K. \& Whitmore, W.F. Jr. Epidemiology of cancer of the prostate. Cancer 1971, 28: 344-360.

2. Byar, D.P. The Veterans Administration Cooperative Urological Research Group's studies of cancer of the prostate. Cancer 1973, 32: 1126-1130.

3. Harrison, G.S.M. The prognosis of prostatic cancer in the younger man. Br J Urol 1983, 55: 315-320.

4. Walsh, P.C. \& Mostwin, J.L. Radical prostatectomy and cystoprostatectomy with preservation of potency. Results using a new nerve-sparing technique. $\mathrm{Br} \mathrm{J}$ Urol 1984, 56: 694-697.

5. Byar, D.P. VACURG studies of post-prostatectomy survival. Scand J Urol Nephrol 1980, 55 (Suppl): 113116.

6. Correa, R.J. Jr., Anderson, R.G., Gibbons, R.P. \& Mason, J.T. Latent carcinoma of the prostate - why the controversy? J Urol 1974, 111: 644-646.

7. Epstein, J.I., Paull, G., Eggleston, J.C. \& Walsh, P.C. Prognosis of untreated stage Al prostatic carcinoma: A study of 94 cases with extended follow up. J Urol 1986, 136: $837-839$.

8. Gibbons, R.P., Correa, R.J. Jr., Brannen, G.E. \& Mason, J.T. Total prostatectomy for localized prostatic cancer. J Urol 1984, 131: 73-76.

9. Elder, J.S., Jewett, H.J. \& Walsh, P.C. Radical perineal prostatectomy for clinical stage B2 carcinoma of the prostate. J Urol 1982, 127: 704-706.

10. Cantril, S.T., Vaeth, J.M., Green, J.P. \& Schroeder, A.F. Radiation therapy for localized carcinoma of the prostate: Correlation with histopathological grading. Front Radiat Ther Oncol 1974, 9: 274.

11. Bagshaw, M.A. Radiotherapy of prostatic cancer. In Pavone-Macaluso, M. \& Smith, P.H. (eds) Cancer of the localized to the prostate especially if it is poorly differentiated and it is unlikely that radical prostatectomy has much to offer the patient if over the age of 70 at the time of diagnosis since the advantages in terms of survival are seen only after ten years. ${ }^{8}$ Many elderly patients are doubtless best left untreated. The management of the younger patient must, however, be different since death is most likely to occur from disseminated prostatic cancer. In such patients radical excision of the prostate is an attractive option.

For those whose disease is beyond the capsule at the time of diagnosis logical evidence in favour of, or against, treatment at first presentation should become available from the results of the MRC trial within the next five years. The outcome of this study may be one of the most important contributions to the management of prostatic cancer within the last forty years.

\section{Acknowledgement}

We are very grateful to Mrs Ann Hopper for her help in the preparation of this paper and for typing the manuscript.

Prostate and Kidney. Plenum Press, New York, London, 1983, pp 283-294.

12. Bloom, H.J.G. Radiotherapy. In Duncan, W. (ed) Recent Results in Cancer Research: Prostate Cancer. Springer-Verlag, Berlin, Heidelberg, New York, 1981, pp 132-153.

13. Paulson, D.F., Lin, G.H., Hinshaw, W., Stephani, S. and the Uro-Oncology Research Group. Radical surgery versus radiotherapy for adenocarcinoma of the prostate. J Urol 1982, 128: 502-504.

14. Paulson, D.F. Treatment of locally confined prostatic cancer: radiotherapy versus surgery; limits of curability. In Kurth, K.H., Debruyne, F.M., Schroeder, F.H. Splinter, T.A.W. \& Wagener, T.D.J. (eds) Progress and Controversies in Oncological Urology. Alan R. Liss, New York, 1984, pp 483-492.

15. Paulson, D.F., Hodge, G.B. Jr, Hinshaw, W. and the Uro-Oncology Research Group. Radiation therapy versus delayed androgen deprivation for stage $\mathrm{C}$ carcinoma of the prostate. J Urol 1984, 131: 901-902.

16. Veterans Administration Co-operative Urological Research Group. Treatment and survival of patients with cancer of the prostate. Surg Gynaecol Obstet 1967, 124: $1011-1017$.

17. Byar, D.P. The Veterans Administration Cooperative Urological Research Group. Bull NY Acad Med 1972, 48: $751-766$.

18. Byar, D.P. (1973) Results of clinical trials of estrogen treatment for cancer of the prostate. In: Proceedings of the 16th Congress of the International Society of Urology, Doin, Paris, 1973, Vol. 2, pp 730-739.

19. Pavone-Macaluso M., Smith P.H., de Voogt H.J. et al. Comparison of diethylstilbestrol, cyproterone acetate and medroxyprogesterone acetate in the treatment of 
advanced prostatic cancer: Final analysis of a randomised phase III trial of the EORTC Urological Group. J Urol 1986, 136: 624-631.

20. Smith P.H., Suciu S., Robinson M.R.G. et al. A comparison of the effect of diethylstilbestrol with low dose estramustine phosphate in the treatment of advanced prostatic cancer: final analysis of a Phase III trial of the FORTC. J Urol 1986, 136: 619-623.

21. Scott W.W. Panel discussion on some aspects of urological endocrinology. In: North Central Section of the American Urological Association Postgraduate Seminar. Burger, Minneapolis, p211. Cited by Thompson G.J. (1959) Long term control of prostatic cancer. Surg Clin North Am 1958, 39: 963-971.

22. Paulson, D.F. Multimodal therapy of prostatic cancer. Urology, 1981, 17: 53.

23. Fair, W.R. Hormonal therapy of advanced prostatic cancer. J Urol 1986, 136: 653-654.

24. Stamey, T.A. Monographs in Urology 1985, 6: 105-18. 